\title{
A new approach to project planning under uncertainty
}

\author{
Nbras Dayoub* and Muhammad Fakhratov \\ Moscow State University of Civil Engineering, 129377, Moscow, Russia
}

\begin{abstract}
This research paper aims to present a dynamic model in the project planning process that is characterized by simplicity and effectiveness, taking into account the internal and external conditions of the project, storing the practical experience and using it later in the subsequent planning processes. The proposed model consists of several entities that have their own independent properties. These entities are linked with each other by bilateral relationships that measure the effect of each one on the other. The proposed model takes into account the different working conditions that may arise during the implementation process. The model has demonstrated possibilities for resource allocation, risk management, forecasting, dynamic planning based on different scenarios, and studying the effectiveness of implementation and the resources usage.
\end{abstract}

\section{Introduction}

Project scheduling is one of the most important problems at the planning and implementation stage of a project. It is a complex issue, given the large number of information and data that must be known before the planning process can begin, and given the variety of methods and models that shape the closest to be the ideal plan, which best uses the resources available for a project, considering the required level of quality, availability of budget and time.

The emergence of the Corona-virus epidemic has changed many concepts that existed before the spread of the epidemic, due to its economic effects in the first place, as many projects have completely stopped, as these economic losses led to the bankruptcy of many companies and thus their closure. $[1,2]$

The project scheduling process aims to develop a plan that defines the start times for the project activities based on the relationships that link these activities with each other and based on the available resources, thus determining the overall project implementation period. The traditional methods depend on considering the plan as a static element $[3,4]$, and the implementation must be strictly adhered to according to this plan, there is a new approach to use the dynamic planning of the project, taking into account the changing conditions of the project during the implementation phase, which is closer to the actual reality. Therefore, in this direction, the implementation plan must be updated continuously. The presence of modern technology and computers enabled a lot of capabilities that were

*Corresponding author: nbrasdayoub@,hotmail.com 
not available before, for example updating the project plan was done over fixed periods of time (daily, weekly, ...), now we can see the impact of any of the factors affecting the project plan In real time, when this factor occurred, that is, the update of the project plan became synchronous and real-time with the internal and external environment of the project $[5,6]$

The lack of data or its unavailability during the planning phase of the project is considered a very important problem. Therefore, some researchers have resorted to considering some project parameters as completely unknown values, such as the activities duration or the resources' productivity (such as workers or machines), or sometimes considering the project implementation is executed in full-uncertainty environment.[7]

Also absent in the studies is the study of the mutual impact of resources with each other during the implementation process, and linking this effect to the conditions under which work is being done.

This research is presented a new practical method that helps the project manager or the responsible person in charge of the implementation in increasing the effectiveness of the production process, identifying the places of weakness or causes that led to the delay and thus addressing them, and at the same time determining the strong sides, to maintain them. This model is not concerned with the nature of the causes or factors (whether internal or external) that affect the execution-process of the project. Rather, it focuses on measuring the percentage of the impact of these factors on the implementation of the plan itself.

This model is based on the dynamic planning of the project, regardless of the method used to obtain the initial plan "baseline", was the Critical Path or PERT for example, where the completion ratios are measured for all partial production operations, in addition to introducing factors based on the estimation of the person responsible for the project planning, and using these results to modify the old plan and getting a more effective new plan which is closer to reality than the old plan.

\section{Materials and Methods}

Assuming we have a project consisting of (n) activities:

$$
A_{1}, A_{2}, \ldots, A_{n}
$$

We have the following resources:

$$
R_{1}, R_{2}, \ldots, R_{n}
$$

The model deals with the project as a database that contains independent entities that have relationships

A separate entity is created for each resource, so that each resource has the following characteristics:

1- Nominal productivity $\left[P_{0}\right]$ : it is the productivity that was adopted during the development of the basic plan.

2- Actual productivity $\left[P_{a}\right]$ : it is the average value of the resource's productivity during the actual work of the resource.

$$
P_{a}=\frac{\sum_{i=1}^{n} P_{a i}}{n}
$$

Where:

$n$ : period of time that presents the duration of the resource

$P_{a i}$ Actual performance on the i-th day 
3- Efficiency coefficient $\left[K_{p}\right]$ : calculated by the following formula:

$$
K_{p}=\frac{P_{a}}{P_{0}}
$$

4- In-team-compatibility coefficient [h]: represents the degree of consistency of the resource in the team in which it works, calculated as follows:

$$
h=\frac{K_{p}-\overline{K_{p}}}{\overline{K_{p}}} \cdot 100
$$

Where: $\overline{K_{p}}$ : Average values of the efficiency coefficient of a resource

5- Workconditions [cw]: This is a descriptive characteristic that describes the situation in which the supplier operates. Where it is equal to the average of the efficiency ratios for all team resources.

Multiple conditions can be added.As the default its value equals to one.

6- Resource evaluation [E]: Calculated by the following formula:

$$
E=h_{i}-h_{i-1}
$$

Where: i represents aday

According to this factor, it can be greater than zero, which means that the resource's performance is improving, or less than zero, which means that the resource's performance is degrading.

The second entity is the team: an organization made up of one or more resources, who work together to produce a specific production unit.

Each team has the following characteristics:

1- Nominal productivity $\left[P_{0}^{T}\right]$ : calculated based on the performance of the resources that make up the team and equal to the lowest present value.

2- Actual productivity $\left[P_{a}^{T}\right]$ : calculated based on the actual productivityof the resources so that it is equal to the minimum present value.

$$
P_{a}^{T}=\min \left(P_{a}^{j}\right)
$$

Where: $P_{a}^{j}$ : Actual productivityof resource $\mathrm{j}$.

3- Efficiency factor $\left[K_{p}^{T}\right]$ :

$$
K_{p}^{T}=\frac{P_{a}^{T}}{P_{0}^{T}}
$$

4- Team Consistency Coefficient $\left[h^{T}\right]$ : This is the value of the standard deviation of the values of the efficiency coefficients of the resources that make up the team.

5- Team Reliability $\left[R^{T}\right]$ : Calculated by the following formula:

$$
R^{T}=B-A
$$

Where B is the average value of the efficiency coefficients greater than zero.

Where $\mathrm{A}$ is the average value of the efficiency coefficients less than zero.

We define a record that contains three fields (Resource - working conditions - value).

Working conditions - a value that expresses the change in the actual productivity of the resource, calculated from the average values of aresource efficiency in a given work condition.Another file is defined to preserve the resources relationships of both with each other.Figure 1 represents the relationships between resources, on the one hand, and with work conditions on the other hand. 


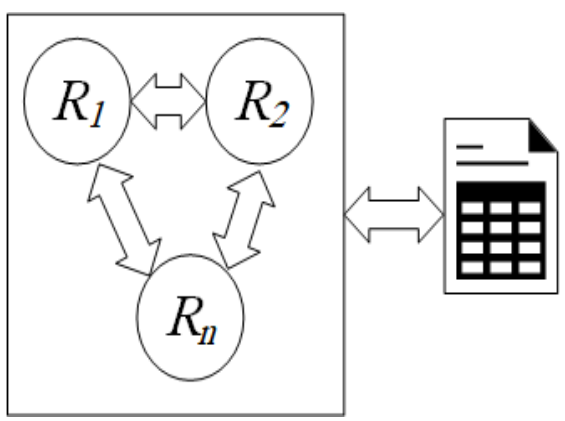

Fig. 1. Resources relationships in the model

The relationship of team resources to each other is determined as follows:

$$
r_{i, j}=\frac{\overline{K_{p l}}+\overline{K_{p J}}}{2}
$$

Where: $\overline{K_{p l}}$ is the average value of the efficiency coefficient for the $\mathrm{i}$ resource in the presence of $\mathrm{j}$ resource.

$\overline{K_{p}}$ the average value of the efficiency coefficient for $\mathrm{j}$ resource in the presence of $\mathrm{i}$ resource.

At a certain point in time (the end of each day or the occurrence of any changes in the working conditions) we apply the following algorithm: 


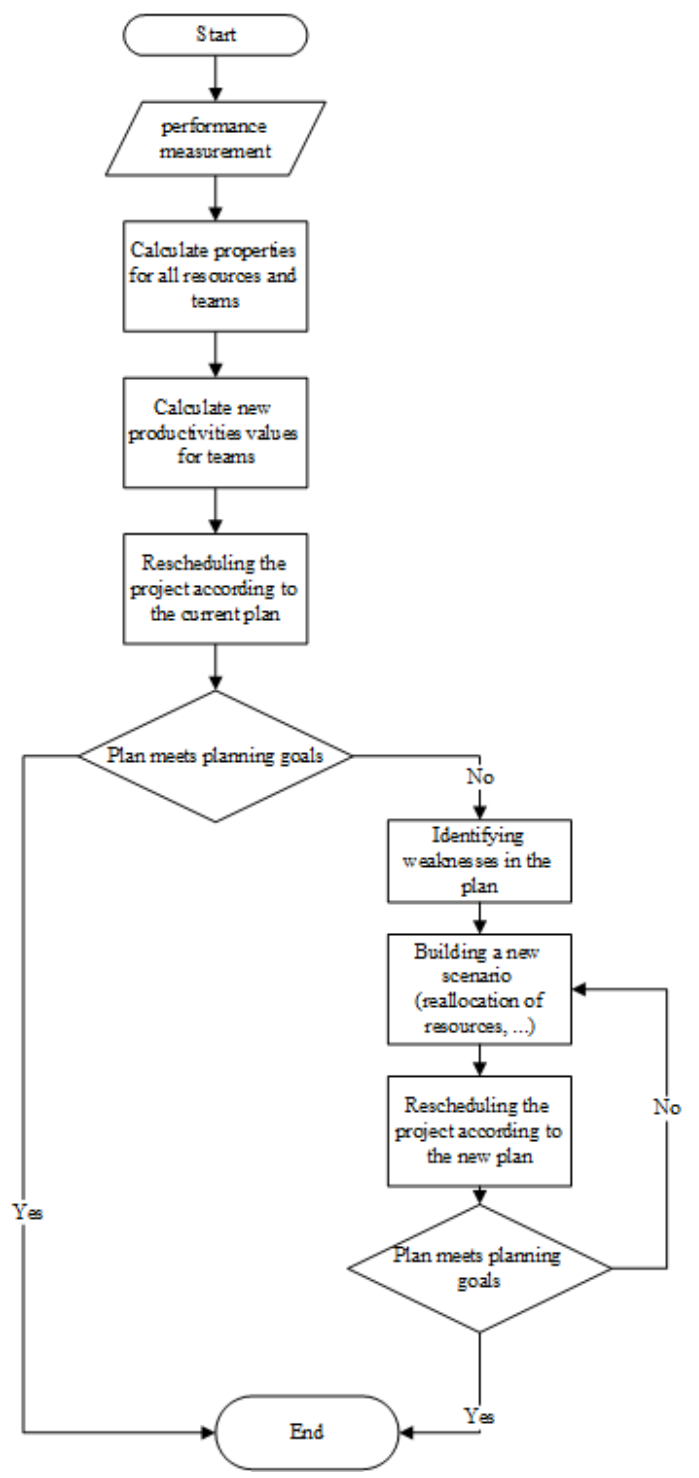

Fig. 2. Decision-making flowchart

In order to clarify the proposed model, the following project was used:

Table 1.Activities properties

\begin{tabular}{llllll}
\hline Activity & \multirow{2}{*}{$\begin{array}{l}\text { Duration } \\
\text { (day) }\end{array}$} & Predecessors & \multicolumn{3}{c}{ Resources need } \\
\cline { 4 - 6 } & & & R1 & R2 & R3 \\
\hline A & 5 & - & 3 & 1 & 1 \\
B & 4 & A & 2 & 2 & 1 \\
C & 3 & A & 2 & 1 & 1 \\
D & 2 & B & 3 & 1 & 1 \\
E & 5 & C & 2 & 2 & 2
\end{tabular}




\begin{tabular}{llllll}
$\mathrm{F}$ & 4 & $\mathrm{D}, \mathrm{E}$ & 3 & 1 & 2 \\
\hline
\end{tabular}

The following resources have been allocated for this project:

Resources of type R1: R11, R12, R13, R14 with a nominal productivity of 5 units of products per day.

Resources of type R2: R21, R22, R23 with a nominal productivity of 4 units of production per day.

Resource type P3: P31, P32 with a nominal productivity of 3 units of production per day.

The project is planned in the critical path method. 'figure3'

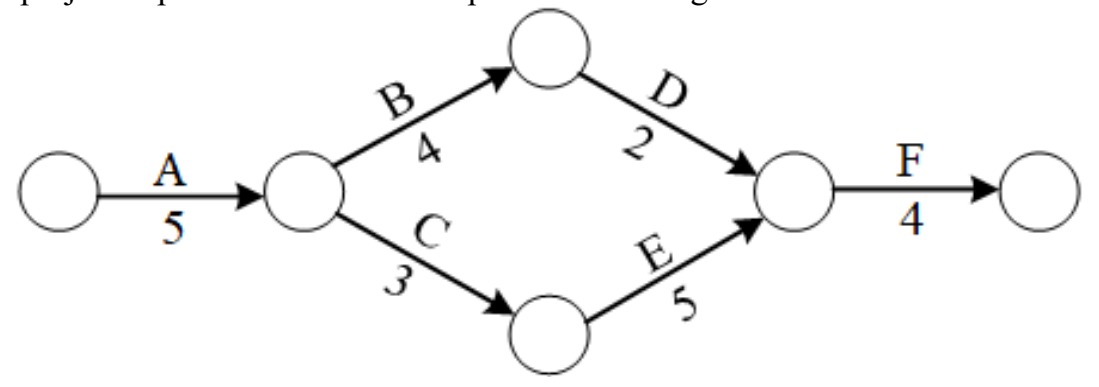

Fig. 3. CPM network diagram

After resource levelling the plane became: 'figure 4'

\begin{tabular}{|c|c|c|c|c|c|c|c|c|c|c|c|c|c|c|c|c|c|c|}
\hline & 1 & 2 & 3 & 4 & 5 & 6 & 7 & 8 & 9 & 10 & 11 & 12 & 13 & 14 & 15 & 16 & 17 & 18 \\
\hline $\mathbf{A}$ & & & & & & & & & & & & & & & & & & \\
\hline B & & & & & & & & & & & & & & & & & & \\
\hline $\mathbf{C}$ & & & & & & & & & & & & & & & & & & \\
\hline D & & & & & & & & & & & & & & & & & & \\
\hline $\mathbf{E}$ & & & & & & & & & & & & & & & & & & \\
\hline $\mathbf{F}$ & & & & & & & & & & & & & & & & & & \\
\hline
\end{tabular}

Fig. 4. Activities schedule of the project

We will apply the approach to the first three tasks, which is the first nine days.

The following T1, T2 and T3 teams have been formed according to the following:

Table 2.Teams properties

\begin{tabular}{rcl}
\hline Team & Activity & Resources \\
\hline T1 & A & R11, R12, R13, R21, R31 \\
T2 & B & R11, R12, R21, R22, R31 \\
T3 & C & R13, R14, R23, R32
\end{tabular}

Let's assume that the implementation these days was carried out on the following values:

Table 3.Daily actual productivity of each resource[unit]

\begin{tabular}{rccccccccc}
\hline Day & R11 & R12 & R13 & R14 & R21 & R22 & R23 & R31 & R32 \\
\hline 1 & 4.51 & 4.03 & 4.08 & - & 3.49 & - & - & 2.89 & - \\
2 & 4.60 & 4.09 & 4.18 & - & 3.45 & - & - & 2.79 & - \\
\hline
\end{tabular}




\begin{tabular}{cccccccccc}
\hline 3 & 4.54 & 4.34 & 4.21 & - & 3.45 & - & - & 2.89 & - \\
4 & 4.48 & 4.21 & 4.28 & - & 3.43 & - & - & 2.77 & - \\
5 & 4.56 & 4.28 & 4.33 & - & 3.46 & - & - & 2.77 & - \\
6 & 4.31 & 4.53 & 4.84 & 4.62 & 3.42 & 3.82 & 3.69 & 2.73 & 2.64 \\
7 & 4.25 & 4.53 & 4.71 & 4.72 & 3.34 & 3.76 & 3.56 & 2.73 & 2.63 \\
8 & 4.22 & 4.49 & 4.95 & 4.69 & 3.42 & 3.73 & 3.63 & 2.76 & 2.63 \\
9 & 4.16 & 4.49 & - & - & 3.36 & 3.79 & - & 2.76 & - \\
\hline
\end{tabular}

\section{Results}

The following results were obtained:

Table 4.Actual productivity values[unit]

\begin{tabular}{rccccccccc}
\hline Day & R11 & R12 & R13 & R14 & R21 & R22 & R23 & R31 & R32 \\
\hline 1 & 4.51 & 4.03 & 4.08 & - & 3.49 & - & - & 2.89 & - \\
2 & 4.56 & 4.06 & 4.13 & - & 3.47 & - & - & 2.84 & - \\
3 & 4.55 & 4.15 & 4.16 & - & 3.46 & - & - & 2.86 & - \\
4 & 4.53 & 4.17 & 4.19 & - & 3.45 & - & - & 2.83 & - \\
5 & 4.54 & 4.19 & 4.22 & - & 3.46 & - & - & 2.82 & - \\
6 & 4.50 & 4.25 & 4.32 & 4.62 & 3.45 & 3.82 & 3.69 & 2.81 & 2.64 \\
7 & 4.47 & 4.29 & 4.38 & 4.67 & 3.43 & 3.79 & 3.63 & 2.80 & 2.63 \\
8 & 4.44 & 4.31 & 4.45 & 4.68 & 3.43 & 3.77 & 3.63 & 2.79 & 2.63 \\
9 & 4.41 & 4.33 & - & - & 3.42 & 3.78 & - & 2.79 & -
\end{tabular}

Table 5.Efficiency coefficient

\begin{tabular}{rccccccccc}
\hline Day & R11 & R12 & R13 & R14 & R21 & R22 & R23 & R31 & R32 \\
\hline 1 & 0.90 & 0.81 & 0.82 & - & 0.87 & - & - & 0.96 & - \\
2 & 0.91 & 0.81 & 0.83 & - & 0.87 & - & - & 0.95 & - \\
3 & 0.91 & 0.83 & 0.83 & - & 0.87 & - & - & 0.95 & - \\
4 & 0.91 & 0.83 & 0.84 & - & 0.86 & - & - & 0.94 & - \\
5 & 0.91 & 0.84 & 0.84 & - & 0.86 & - & - & 0.94 & - \\
6 & 0.90 & 0.85 & 0.86 & 0.92 & 0.86 & 0.95 & 0.92 & 0.94 & 0.88 \\
7 & 0.89 & 0.86 & 0.88 & 0.93 & 0.86 & 0.95 & 0.91 & 0.93 & 0.88 \\
8 & 0.89 & 0.86 & 0.89 & 0.94 & 0.86 & 0.94 & 0.91 & 0.93 & 0.88 \\
9 & 0.88 & 0.87 & - & - & 0.86 & 0.94 & - & 0.93 & -
\end{tabular}

Table 6.In-team-compatibility coefficient

\begin{tabular}{rccccccccc}
\hline Day & R11 & R12 & R13 & R14 & R21 & R22 & R23 & R31 & R32 \\
\hline 1 & 3.51 & -7.62 & -6.37 & - & 0.03 & - & - & 10.45 & - \\
2 & 4.45 & -7.00 & -5.34 & - & -0.62 & - & - & 8.51 & - \\
\hline
\end{tabular}




\begin{tabular}{cccccccccc}
\hline 3 & 3.68 & -5.41 & -5.31 & - & -1.39 & - & - & 8.43 & - \\
4 & 3.39 & -5.01 & -4.50 & - & -1.55 & - & - & 7.68 & - \\
5 & 3.33 & -4.66 & -4.01 & - & -1.68 & - & - & 7.02 & - \\
6 & -0.01 & -5.70 & -3.74 & 2.88 & -4.21 & 6.03 & 2.85 & 3.89 & -2.00 \\
7 & -0.48 & -4.50 & -2.57 & 3.95 & -4.35 & 5.52 & 0.94 & 3.82 & -2.32 \\
8 & -1.00 & -3.77 & -1.43 & 3.65 & -4.25 & 5.19 & 0.53 & 3.82 & -2.75 \\
9 & -1.59 & -3.24 & - & - & -4.37 & 5.42 & - & 3.78 & -
\end{tabular}

Table 7.Resource evaluation

\begin{tabular}{rccccccccc}
\hline Day & R11 & R12 & R13 & R14 & R21 & R22 & R23 & R31 & R32 \\
\hline 1 & - & - & - & - & - & - & - & - & - \\
2 & 0.94 & 0.62 & 1.03 & - & -0.66 & - & - & -1.93 & - \\
3 & -0.77 & 1.59 & 0.03 & - & -0.76 & - & - & -0.09 & - \\
4 & -0.30 & 0.40 & 0.81 & - & -0.17 & - & - & -0.75 & - \\
5 & -0.05 & 0.35 & 0.49 & - & -0.13 & - & - & -0.66 & - \\
6 & -3.35 & -1.04 & 0.28 & - & -2.53 & - & - & -3.13 & - \\
7 & -0.47 & 1.20 & 1.17 & 1.07 & -0.14 & -0.51 & -1.91 & -0.08 & -0.32 \\
8 & -0.52 & 0.73 & 1.13 & -0.30 & 0.10 & -0.33 & -0.41 & 0.01 & -0.42 \\
9 & -0.59 & 0.53 & - & - & -0.13 & 0.23 & - & -0.04 & -
\end{tabular}

Table 8.T1 Team properties

\begin{tabular}{cccccc} 
Day & $P_{0}^{T}$ & $P_{a}^{T}[$ unit $]$ & $K_{p}^{T}$ & $h^{T}[\%]$ & $R^{T}$ \\
\hline 1 & 5 & 4.03 & 0.81 & 5.79 & \\
2 & 5 & 4.06 & 0.81 & 5.09 & -0.43 \\
3 & 5 & 4.15 & 0.83 & 4.71 & 0.27 \\
4 & 5 & 4.17 & 0.83 & 4.26 & 0.20 \\
5 & 5 & 4.19 & 0.84 & 3.95 & 0.14 \\
\hline
\end{tabular}

Table 10. R11 Resource relationships values with other resources

\begin{tabular}{ccccccc} 
Day & $\mathrm{r}_{11,12}$ & $\mathrm{r}_{11,13}$ & $\mathrm{r}_{11,21}$ & $\mathrm{r}_{11,31}$ & $\mathrm{r}_{11,32}$ & $\mathrm{r}_{11,22}$ \\
\hline 1 & 0.85 & 0.86 & 0.89 & 0.93 & & \\
2 & 0.86 & 0.87 & 0.89 & 0.93 & & \\
3 & 0.87 & 0.87 & 0.89 & 0.93 & & \\
4 & 0.87 & 0.87 & 0.89 & 0.93 & & \\
5 & 0.87 & 0.88 & 0.89 & 0.92 & & \\
6 & 0.87 & & 0.88 & 0.92 & 0.89 & 0.93 \\
7 & 0.88 & & 0.88 & 0.91 & 0.89 & 0.92 \\
8 & 0.87 & & 0.87 & 0.91 & 0.88 & 0.91 \\
9 & 0.87 & & 0.87 & 0.91 & 0.88 & 0.91 \\
\hline
\end{tabular}




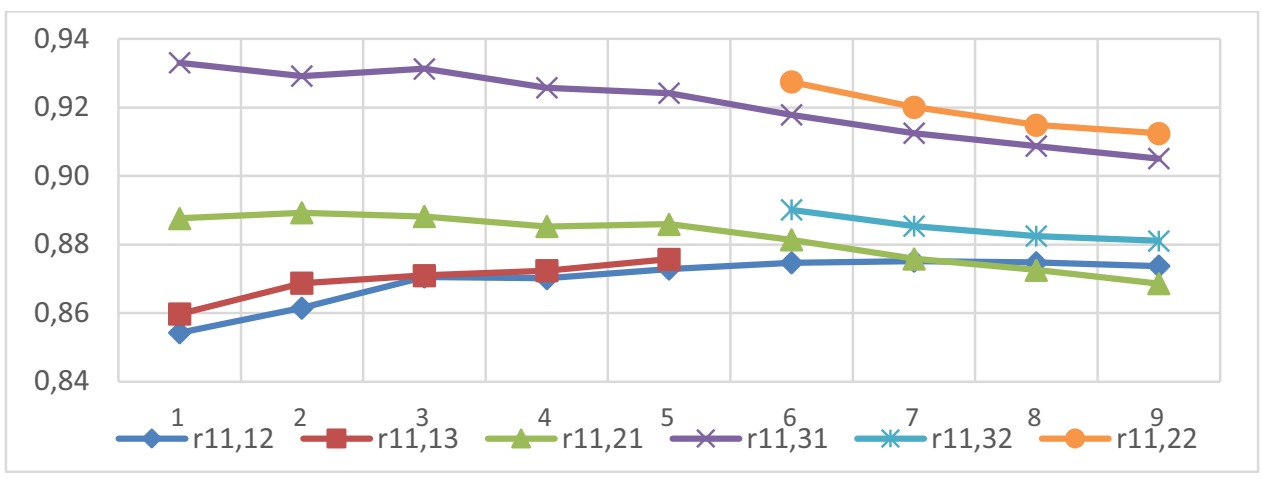

Fig. 5. R11 relationships with other resources

\section{Discussion}

This method is simple, but provides effective results.

This model offers the following capabilities:

1- Dynamism in planning: as the plan update can take place either according to specific periods of time or immediately when the change occurs, and this is the closest representation of reality, and because of the uncertainty that characterizes projects in real life, resulting from the great multiplicity of internal and external factors that Affects directly or indirectly the project implementation process.

2- Effective risk management by monitoring the changes that occur according to several levels (resource, team, activity, ...) and the reasons that led to these changes. This measurement is done digitally, so that every "cause" is expressed with its direct impact on theproject flow process.

3- Allocating resources and evaluating them through the bilateral relations that are created every day (unit of time), and thus resources can be re-allocated so that they form different teams according to a specific goal (such as the greatest consensus, for example) or assigning teams that have proven their effectiveness to critical tasks that directly affect. Over the duration of the project, this approach also allows preserving the practical experience of each resource separately according to different working conditions, and thus the possibility of distributing resources more effectively during future planning processes.

4- Scenarios and forecasting thanks to the existence of actual past information that expresses the actual productivity of a particular resource on the one hand, and by linking changing working conditions on the other hand.

5- Ease of programming with the presence of modern technology, it has become easy to develop software based on the previous model with integration with mobile devices applications for teams of workers or quality controllers at the work site, and linking all this information according to a local database (related to the project) or central (related to the company's projects as a whole).

6- Having information in one place, the method's reliance on databases does not give the opportunity to lose or duplicate information, but attention must be paid to the users' permissionswho are able to access and modify this data.

7- Taking into consideration individual behaviors and the effect they bite on each other as part of the actual reality, where it is possible that there are people who do not have enough experience, or that some workers do not cooperate with each other for one reason or another, the model provides the possibility of measuring these effects and their reflection 
on the productivity of the team as a whole. Therefore, identifying the places of weakness and identifying its causes.

8- Flexibility and possibility of development This model can be developed in several areas, for example bilateral relations of resources allow the possibility of forming teams at high rates of production with a certain probability, here we must take advantage and develop a model that allows the optimal use of these relationships.

9- Moving away from personal opinions in evaluating resources in the model. All factors are calculated based on measured values, so that as much as possible, personal opinions are avoided and fairness is achieved in evaluating project resources.

10- The accumulation of experience. The information taken from the project is preserved (whether it is the productivity of the resource or working conditions ...) it is saved in a way that allows it to be used again in future planning.

Application limits, this model gives wide flexibility in the application, it can be applied at both the company level (several projects), or at the level of the project itself. Its correct application requires the presence of a follow-up team that carries out its tasks responsibly and accurately, to ensure that results are actually expressed in reality.

This paper presented this model with its main ideas, and certainly the research must be pursued and some points studied, such as allocating resources automatically or according to a specific model that allows the project manager to develop several scenarios and choose the appropriate according to the goals he deems appropriate at a specific stage of project implementation.

\section{Conclusions}

Modern technology and database programming provided many new possibilities, which necessitated the development of methods and models for problems related to project planning and other issues related to planning and management processes. This research suggested a model in its basic form. Further research should be conducted to find the most effective method, which at the same time meets the objectives of the planning phase.

\section{References}

1. F. Hao, Q. Xiao and K. Chon, COVID-19 and China's Hotel Industry: Impacts, a Disaster Management Framework, and Post-Pandemic Agenda, Int. J. Hosp. Manag. 90 (2020)

2. E. Forsythe, L.B. Kahn, F. Lange and D. Wiczer, Labor demand in the time of COVID-19: Evidence from vacancy postings and UI claims, J. Public Econ.189, 104238 (2020)

3. D. Trietsch and K.R. Baker, PERT 21: Fitting PERT/CPM for use in the 21st century Int. J. Proj. Manag. 30, 490-502 (2012)

4. Y-R Wang, C-Y Yu and H-H Chan, Predicting construction cost and schedule success using artificial neural networks ensemble and support vector machines classification models, Int. J. Proj. Manag. 30,470-8 2012

5. T. Kim, Y. Kim and H. Cho, Dynamic production scheduling model under due date uncertainty in precast concrete construction, J. Clean. Prod. 257, 120527 (2020)

6. L. Cadarso and R. de Celis, Integrated airline planning: Robust update of scheduling and fleet balancing under demand uncertainty, Transp. Res. Part C Emerg. Technol. 81 $227-45$ (2017) 
7. O. Lambrechts, E. Demeulemeester and W. Herroelen, Proactive and reactive strategies for resource-constrained project scheduling with uncertain resource availabilities, J. Sched. 11, 121-36 (2008)

8. F. Deblaere, E. Demeulemeester and W. Herroelen, Reactive scheduling in the multimode RCPSP, Comput. Oper. Res. 38, 63-74 (2011) 